Document downloaded from:

http://hdl.handle.net/10251/82088

This paper must be cited as:

Gómez Barquero, D.; Vargas, D.; Fuentes Muela, M.; Klenner, P.; Moon, S.; Choi, J.; Schneider, D.... (2016). MIMO for ATSC 3.0. IEEE Transactions on Broadcasting. 62(1):298305. doi:10.1109/TBC.2015.2505399.

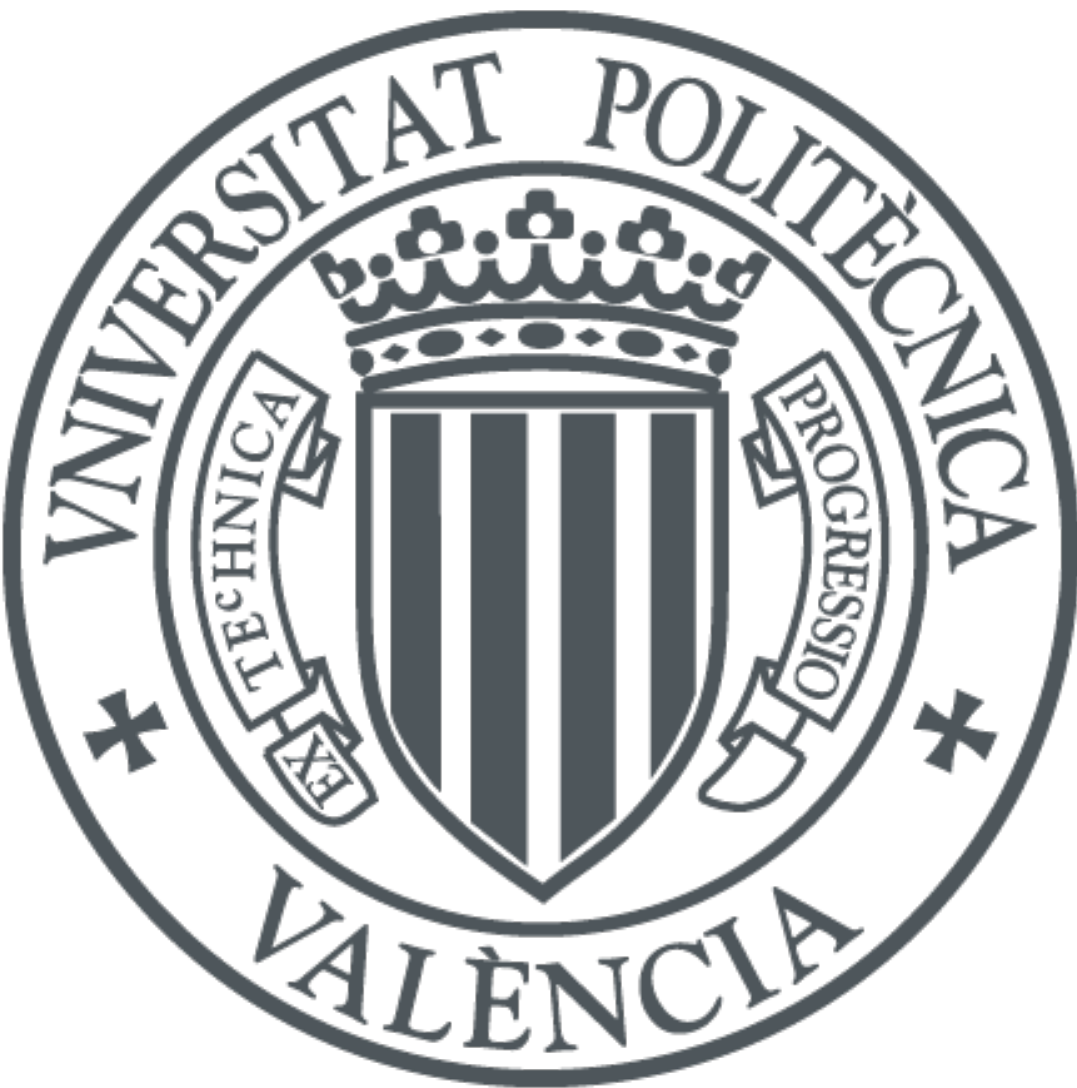

The final publication is available at

http://dx.doi.org/10.1109/TBC.2015.2505399

Copyright Institute of Electrical and Electronics Engineers (IEEE)

Additional Information

"(c) 2016 IEEE. Personal use of this material is permitted. Permission from IEEE must be obtained for all other users, including reprinting/ republishing this material for advertising or promotional purposes, creating new collective works for resale or redistribution to servers or lists, or reuse of any copyrighted components of this work in other works.") 


\title{
MIMO for ATSC 3.0
}

\author{
David Gómez-Barquero, David Vargas, Manuel Fuentes, Peter Klenner, Sangchul Moon, \\ Jin-Yong Choi, Daniel Schneider, and Kenichi Murayama
}

\begin{abstract}
This paper provides an overview of the optional MIMO (Multiple-Input Multiple-Output) antenna scheme adopted in ATSC 3.0 to improve robustness or increase capacity via additional spatial diversity and multiplexing by sending two data streams in a single radio frequency channel. Although it is not directly specified, it is expected in practice to use cross-polarized $2 \times 2$ MIMO (i.e., horizontal and vertical polarization) to retain multiplexing capabilities in line-of-sight conditions. MIMO allows overcoming the channel capacity limit of single antenna wireless communications in a given channel bandwidth without any increase in the total transmission power. But in the U.S. MIMO can actually provide a larger comparative gain because it would be allowed to increase the total transmit power, by transmitting the nominal transmit power in each polarization. Hence, in addition to the MIMO gains (array, diversity and spatial multiplexing), MIMO could exploit an additional $3 \mathrm{~dB}$ power gain. The MIMO scheme adopted in ATSC 3.0 re-uses the SISO (SingleInput Single-Output) antenna baseline constellations, and hence it introduces the use of MIMO with non-uniform constellations.
\end{abstract}

Index Terms-ATSC 3.0, MIMO, DTT, spatial multiplexing.

\section{INTRODUCTION}

$\mathrm{M}$ IMO (Multiple-Input Multiple-Output) antenna technology has been introduced in many wireless communications technologies such as WiFi, WiMAX and $4 \mathrm{G}$ LTE to improve the transmission robustness via additional spatial diversity, or to increase capacity by sending multiple data streams in the same bandwidth via spatial multiplexing [1]. While SIMO (Single-Input Multiple-Output) exploits diversity and array gains, MISO (Multiple-Input Single-Output) only retains the spatial diversity gain. The spatial multiplexing gain is achieved only in the case of MIMO, and it allows MIMO to overcome the capacity limit of single transmit antenna wireless communications in a given channel bandwidth without any increase in the total transmission power [2].

Terrestrial MIMO broadcasting in the UHF (Ultra-High Frequency) band requires co-located ${ }^{1}$ antennas with cross-polar (horizontal and vertical) polarization to retain full spatial multiplexing capabilities in line-of-sight conditions [3].

Manuscript received August 7, 2015; reviewed October 12, 2015.

D. Gómez-Barquero, D. Vargas, and M. Fuentes are with the Insitute of Telecommunications and Multimedia Applications (iTEAM), Universitat Politecnica de Valencia, Valencia 46022, Spain (e-mail: \{dagobar,davarpa,mafuemue\}@iteam.upv.es)

P. Klenner is with Panasonic Europe, Langen 63225, Germany (e-mail: peter.klenner@eu.panasonic.com)

S. Moon and J.-Y. Choi are with LG Electronics, Seoul 150-721, Korea (email: \{sangchul.moon, jinyong99.choi\}@lge.com).
Another important difference of terrestrial MIMO broadcasting compared to unicast wireless communications systems is that it is not possible to share the Channel State Information (CSI) of the receivers with the transmitter, which reduces the potential gain. Nevertheless, the potential MIMO gain in terrestrial broadcasting is still very important, especially for medium and high signal-to-noise ratios (SNR) [4]. Also, the channel precoder may be optimized according to broadcast channel statistics by linearly combining the data streams across the transmit antennas [5].

MIMO for terrestrial broadcast has been demonstrated in field tests by the public broadcasters BBC (UK) [6] and NHK (Japan) [7]. The DVB-NGH technical specification [8] was the first terrestrial broadcasting standard that allows for the optional use of MIMO [9], although the technology has not been yet proved in the field, and field measurements were used to develop representative channel models for pedestrian outdoor, indoor, and mobile reception [4].

This paper provides an overview of the optional MIMO antenna system adopted in ATSC 3.0 intended for $2 \times 2$ crosspolarized MIMO [10]. This means that at least two antenna aerials with horizontal and vertical polarization are present at both transmitter and receiver side. The transmitter must include individually-fed cross-polar antennas, and the receiver must include a cross-polar pair of antennas and two tuners in order to receive and decode the MIMO signal.

MIMO may become a key technology for ATSC 3.0 because the current commercial state-of-the-art digital terrestrial television (DTT) standard, DVB-T2 [11], does not include this feature. Considering the relevance of maximizing the broadcast spectrum efficiency due to the rapidly growing demand for wireless broadband services [12], MIMO is the most convincing technology that brings huge benefit in this aspect. Furthermore, in the U.S. MIMO can actually provide a larger comparative gain because the regulation allows to increase the total transmit power by transmitting the nominal transmit power in each polarization. Hence, in addition to the MIMO gain, it could exploit a $3 \mathrm{~dB}$ power gain. MIMO is also an effective way to impose the use of two antennas at the receivers, which results on average in another $3 \mathrm{~dB}$ gain compared to SISO for crosspolarized transmissions.

D. Schneider is with Sony Deutschland GmbH, 70327 Stuttgart, Germany (daniel.schneider@eu.sony.com).

K. Murayama is with NHK Science \& Technology Research Laboratories, Tokyo 157-8510, Japan (e-mail: murayama.k-fu@nhk.or.jp).

${ }^{1}$ It should be pointed out that MIMO with two separately located antennas spanned across two broadcast towers is a MISO Single Frequency Network (SFN) with receivers with two antennas and a single demodulator, and cannot exploit spatial multiplexing due to the resulting power imbalances. 

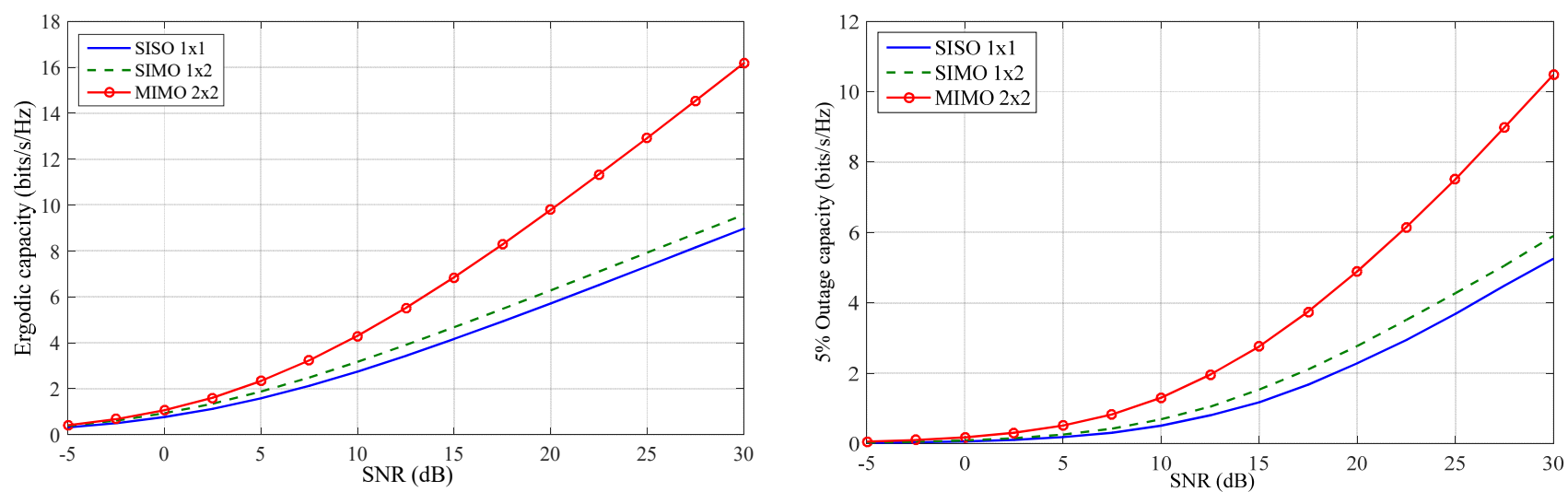

Fig. 1. Ergodic capacity for a mobile DVB-NGH channel model (left) and $5 \%$ outage capacity for fixed rooftop MGM channel model (right). The fixed rooftop model includes a $9 \mathrm{~dB}$ lognormal fading margin in the CNR. This means that SNRs in the MGM channel (right) are $9 \mathrm{~dB}$ higher than the required SNR for the system modulation and coding, which correspond to the SNR-axis in the DVB-NGH channel (left). SNR values of 24 and $27 \mathrm{~dB}$ correspond to SNR thresholds of $15 \mathrm{~dB}$ and $18 \mathrm{~dB}$, respectively.

The rest of the paper is structured as follows. Section II provides some basics about the MIMO channel capacity gain and implementation aspects. Section III describes the MIMO transmission chain adopted in ATSC 3.0. Section IV provides illustrative performance simulation results. The paper is concluded in Section V, where a brief discussion about the potential introduction of MIMO in the U.S. is given.

\section{MIMO BASICS}

\section{A. MIMO Gain}

The implementation of MIMO provides three different types of gains: array gain, diversity gain, and multiplexing gain [9]. The array gain is due to the coherent combination of the received signals with several antennas (for co-polar antennas, this gain equals $3 \mathrm{~dB}$ every time the number of antennas is doubled). The diversity gain is due to the fact of having several spatial branches (with independent fading) between the transmitter and receiver. And the multiplexing gain allows transmitting more than one data stream.

Fig. 1 shows the channel capacity for SISO, SIMO with two receive antennas, and MIMO with two transmit and two receive antennas, in the mobile DVB-NGH channel [4] (user speed $60 \mathrm{~km} / \mathrm{h}$ ) with ergodic capacity and the modified Guildford channel model (MGM) from BBC for fixed rooftop reception $[3,6,13]$ with $5 \%$ outage capacity. Both models have been specifically developed for terrestrial broadcasting based on field measurements. While ergodic capacity is an appropriate metric for mobile broadcasting applications, the $\varepsilon$-outage capacity [2] is more suited for fixed broadcasting applications. The outage capacity can be mathematically expressed as:

$C_{\varepsilon} \cong \max \left\{R \mid \operatorname{Pr}\left(C_{\mathbf{H}}<R\right) \leq \varepsilon\right\}$

where $C_{\mathbf{H}}$ is the capacity of a specific channel realization and $\operatorname{Pr}\left(C_{\mathbf{H}}<R\right)$ is the probability that $C_{\mathbf{H}}$ is lower than the rate $R$. The $\varepsilon$-outage capacity in (1) can be interpreted as the minimum rate $C_{\varepsilon}$ that can be achieved the $(1-\varepsilon) \cdot 100 \%$ of the channel realizations, or in the specific case of the MGM of the receiving population.

In Fig. 1, it can be seen that the MIMO capacity increases with the SNR at a higher rate than SISO and SIMO schemes.
This difference in spectral efficiency increases with increasing SNR and is due to multiplexing gain for a MIMO channel employing multiple transmit and receive antennas with independent information streams. The gain depends also on the particular channel. The predicted MIMO benefit over SISO for the fixed rooftop model is about $100 \%$ for SNRs ranging between 24 and $27 \mathrm{~dB}$. Small portable devices with built-in antennas might not get as much received signal strength. However, increased diversity due to multiple antennas at low SNR levels provides very significant gains in relative terms. In particular, the DVB-NGH mobile model gives a gain of $40 \%$, $48 \%$ and $57 \%$, for 0,5 and $10 \mathrm{~dB}$ SNR, respectively.

Regarding the additional gain that may be achieved by doubling the total transmit power with MIMO, the BBC fixed channel model yields an extra $50 \%$ gain due to the $3 \mathrm{~dB}$ power increase for a SISO SNR of $24 \mathrm{~dB}$ ( $40 \%$ for $27 \mathrm{~dB}$ SNR). For mobile reception, the gain increases in $92 \%, 71 \%$ and $54 \%$, for 0,5 and $10 \mathrm{~dB}$ SISO SNR, respectively.

Finally, it should be pointed out that MIMO requires the use of twice the pilots compared to SISO for the same performance in terms of echo tolerance and Doppler [15]. Hence, the additional pilot overhead should be taken into account to derive the final gain.

\section{B. MIMO Implementation}

There are important implementation implications on both transmit and receive sides when deploying MIMO.

For the transmitter side, there are: (i) new antenna(s), (ii) additional cabling, and (iii) second transmit data stream (amplifier, up-converter, etc.). The modulator outputs two baseband signals (one for each antenna), and then the whole RF chain needs to be doubled. If MIMO is used to increase the total transmission power, it would naturally imply additional capital and operational costs.

For the receiver the implications are: (i) cross-polarized antennas, (ii) two tuners, and (iii) higher constellation demapper complexity [16]. If MIMO is used to increase the overall system capacity, the receiver naturally has to support the increased peak data rates. It should be pointed out that a receiver with two tuners supporting channel bonding [17] would pave the way for advanced MIMO receivers, since a channel bonding receiver requires two tuners and the capability of supporting 


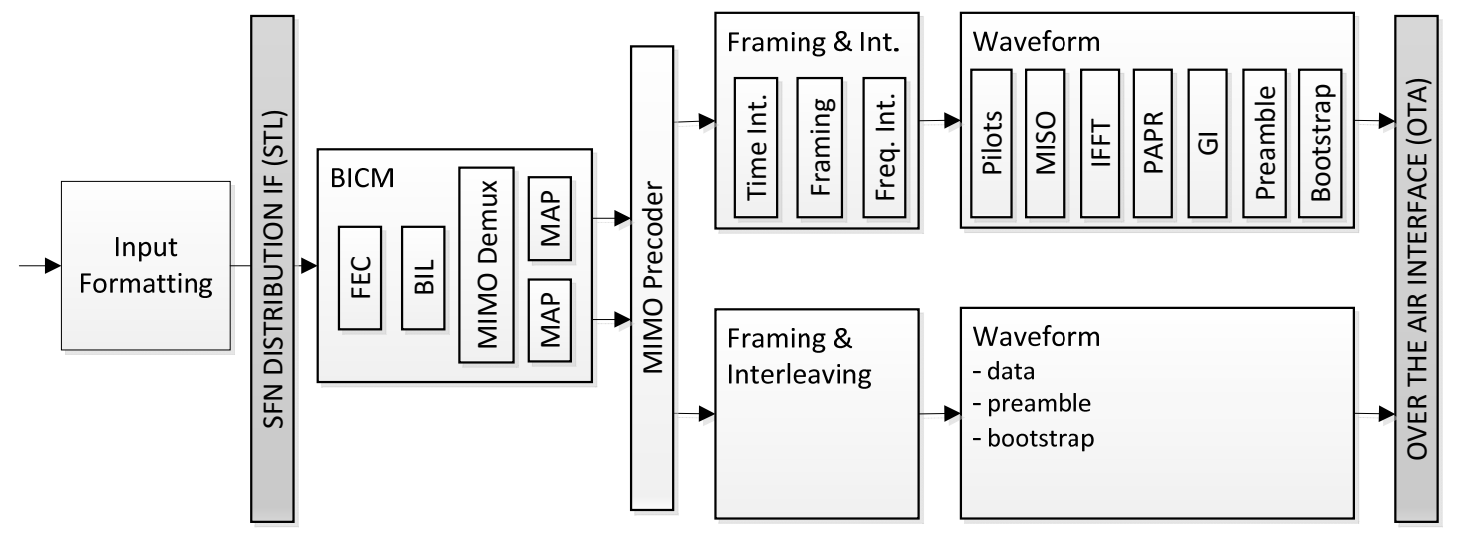

Fig. 2. MIMO transmit block diagram in ATSC 3.0. Acronyms: IF (interface), STL (studio transmitter link), FEC (forward error correction), BIL (bit interleaver), MIMO Demux (MIMO antenna stream demultiplexer), MAP (constellation mapper), MISO (Multiple-Input Single-Output), PAPR (Peak-to-Average Power Ratio), GI (Guard Interval).

increased (in particular, doubled) peak data rates. The implementation of MIMO for fixed rooftop reception also requires that the receiver installation accommodates the reception of the two streams to the demodulator, which may require substantial changes in existing installations based on single antenna reception.

\section{ATSC 3.0 MIMO SYSTEM OVERVIEW}

Fig. 2 depicts the ATSC 3.0 MIMO transmission chain, where two MIMO blocks can be identified: the MIMO demultiplexer and the MIMO precoder. Naturally, all blocks, which are carried out after the generation of the two MIMO streams in the MIMO demultiplexer, are doubled, one block for each antenna (e.g., constellation mapper, framing and interleaving, and waveform generation).

The MIMO transmission chain re-uses all the blocks from the SISO baseline [10], including forward error correction (FEC) codes, bit interleavers (BIL), constellations ${ }^{2}$, frequency and time interleavers, etc. The same time and frequency interleaving configuration should be applied to both MIMO streams. The only two differences compared to the SISO baseline are that specific pilot patterns have been defined for MIMO, and that MIMO requires twice the time interleaver memory as SISO. The MIMO pilot patterns achieve the same performance in terms of echo tolerance and Doppler as for SISO. The doubling of the time interleaving memory is due to the fact that the SISO memory requirement applies to the memory of each antenna, providing the same time interleaving trade-off as for SISO.

MIMO processing in ATSC 3.0 is only applicable to the data path, and it is not applied to the bootstrap, which is the entry point to the ATSC 3.0 system, and to the preamble with layer1 (L1) signaling information [19]. The use of MIMO is signaled in the preamble. The use of mixed SISO and MIMO frames in the same RF channel is possible in ATSC 3.0, and it is also signaled in the preamble.

The use of MIMO with channel bonding [17], constellation superposition (Layered Division Multiplexing, LDM) [20], and the peak-to-average power ratio (PAPR) reduction technique

2 The MIMO scheme adopted in ATSC 3.0 re-uses the SISO antenna baseline constellations, and hence it introduces the use of MIMO with NonUniform Constellations (NUC). In addition to QPSK, ATSC 3.0 has adopted

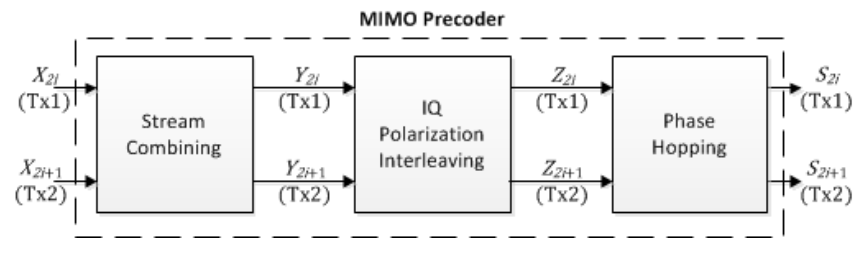

Fig. 3. ATSC 3.0 MIMO precoder sub-block diagram consisting of stream combination, IQ polarization interleaving and phase hopping.

ACE (Active Constellation Extension) is not defined in the ATSC 3.0 specification. The use of MISO with Transmit Diversity Code Filter Sets (TDCFS) may be optionally applied with a unique code applied per station or per-antenna [21].

\section{A. MIMO Demultiplex}

The antenna stream demultiplexer distributes the output bits from the bit interleaver into two constellation mappers, one for each transmit antenna. The output bits are alternatively distributed to the mappers in groups, and the group size corresponds to the number of bits per constellation symbol (cell). That is, the demultiplexer is equivalent to an even/odd cell demultiplexer but implemented at the bit level.

\section{B. MIMO Precoder}

Fig. 3 illustrates the MIMO precoder, which is based on Spatial Multiplexing (SM) and consists of three different steps:

- Stream combining.

- I/Q polarization interleaving.

- Phase hopping.

The MIMO precoder acts on a pair of input constellation symbols $\left(X_{2 i}, X_{2 i+1}\right)$, where $i$ is the index of the cell pair within the FEC codeword, and creates a pair of outputs constellation symbols $\left(S_{2 i}, S_{2 i+1}\right)$. Encoded cell pairs are transmitted on the same OFDM symbol and carrier from transmitter \#1 (Tx1) and transmitter \#2 (Tx2), respectively.

The MIMO precoding is applied at data physical layer pipe (PLP) level, and each of the three sub-blocks of the MIMO precoder can be optionally activated (and their use is signaling in the L1). This allows the possibility of configuring the precoder in a transparent way in the transmission chain, such

two-dimensional NUC (2D-NUC) for 16-, 64- and 256-point constellations, and one-dimensional NUCs (1D-NUC) for 1024- (1k) and 4096-point (4k) constellations [18]. 
TABLE I

RotATION ANGLE FOR THE STREAM COMBINING OF THE MIMO PRECODER

\begin{tabular}{c|ccc}
\hline \hline $\begin{array}{c}\text { Coding } \\
\text { Rate }\end{array}$ & QPSK & 16QAM & $\begin{array}{c}\text { 64QAM, 256AM, } \\
\text { 1024QAM,4086QAM }\end{array}$ \\
\hline $2 / 15$ & $0^{\circ}$ & $0^{\circ}$ & $0^{\circ}$ \\
$3 / 15$ & $0^{\circ}$ & $0^{\circ}$ & $0^{\circ}$ \\
$4 / 15$ & $0^{\circ}$ & $0^{\circ}$ & $0^{\circ}$ \\
$5 / 15$ & $0^{\circ}$ & $0^{\circ}$ & $0^{\circ}$ \\
$6 / 15$ & $15^{\circ}$ & $0^{\circ}$ & $0^{\circ}$ \\
$7 / 15$ & $15^{\circ}$ & $0^{\circ}$ & $0^{\circ}$ \\
$8 / 15$ & $20^{\circ}$ & $0^{\circ}$ & $0^{\circ}$ \\
$9 / 15$ & $20^{\circ}$ & $0^{\circ}$ & $0^{\circ}$ \\
$10 / 15$ & $25^{\circ}$ & $0^{\circ}$ & $0^{\circ}$ \\
$11 / 15$ & $25^{\circ}$ & $15^{\circ}$ & $0^{\circ}$ \\
$12 / 15$ & $25^{\circ}$ & $15^{\circ}$ & $0^{\circ}$ \\
$13 / 15$ & $30^{\circ}$ & $15^{\circ}$ & $0^{\circ}$ \\
\hline \hline
\end{tabular}

that the output pair cells are exactly the input pair cells. This particular case is known as plain spatial multiplexing.

$S_{2 i}(\mathrm{Tx} 1)=Z_{2 i}(\mathrm{Tx} 1)=Y_{2 i}(\mathrm{Tx} 1)=X_{2 i}(\mathrm{Tx} 1)$

$S_{2 i+1}(\mathrm{Tx} 2)=Z_{2 i+1}(\mathrm{Tx} 2)=Y_{2 i+1}(\mathrm{Tx} 2)=X_{2 i+1}(\mathrm{Tx} 2)$

The next sub-sections describe the signal processing performed at each sub-block of the precoder when they are used. If not used, the output pair cells are exactly the input pair cells.

\section{1) Antenna Stream Combination}

The stream combining consists on a linear combination of the pair of input constellation symbols based on a rotation matrix with angle $\theta$. It improves the spatial diversity under spatially correlated channels because symbols are not transmitted only from one of the transmitted antennas [2]. The presence of correlation in the MIMO terrestrial broadcast channel is common in line-of-sight conditions [26]. The scheme adopted in ATSC 3.0 is similar to the precoder adopted in DVB-NGH [22]. The operation is mathematically expressed as follows:

$\left[\begin{array}{c}Y_{2 i}(\mathrm{Tx} 1) \\ Y_{2 i+1}(\mathrm{Tx} 2)\end{array}\right]=\left[\begin{array}{cc}\cos \theta & \sin \theta \\ \sin \theta & -\cos \theta\end{array}\right]\left[\begin{array}{c}X_{2 i}(\mathrm{Tx} 1) \\ X_{2 i+1}(\mathrm{Tx} 2)\end{array}\right]$

The value of the rotation angle $\theta$ is fixed and its value depends on the modulation and coding (ModCod) used in the PLP.

Table I shows the rotation angle $\theta$ for each coding rate for QPSK and 16QAM. The rotation angles have been selected to improve the performance in scenarios where there is a power imbalance (PI) between the receive antennas. This imbalance could be produced at the transmit side, but also due to the channel impairments. In the performance evaluations, no gain was observed for higher order constellations due to rotation. It should also be pointed out that if there is no power imbalance, the optimum rotation angle is $0^{\circ}$ for all ModCods.

\section{2) I/Q Polarization Interleaving}

The I/Q polarization interleaver is simply a switching interleaving operation, such that the output cells consist of the real (In-phase) component of one input symbol and the imaginary (Quadrature) component of the other input symbol. The I/Q polarization interleaving is described by the following equations:

$$
\begin{aligned}
& Z_{2 i}(\mathrm{Tx} 1)=\operatorname{Re}\left\{Y_{2 i}(\mathrm{Tx} 1)\right\}+j \cdot \operatorname{Im}\left\{Y_{2 i+1}(\mathrm{Tx} 2)\right\} \\
& Z_{2 i+1}(\mathrm{Tx} 2)=\operatorname{Re}\left\{Y_{2 i+1}(\mathrm{Tx} 2)\right\}+j \cdot \operatorname{Im}\left\{Y_{2 i}(\mathrm{Tx} 1)\right\}
\end{aligned}
$$

TABLE II

MIMO SCATTERED PILOT SCHEMES AND EQUIVALENT SISO PILOT SCHEMES

\begin{tabular}{c|cccc}
\hline $\begin{array}{c}\text { MIMO } \\
\text { pilot scheme }\end{array}$ & $D_{\mathrm{X}}$ & $D_{\mathrm{Y}}$ & $\begin{array}{c}\text { Equivalent } \\
\text { SISO pilot } \\
\text { scheme }\end{array}$ & Overhead \\
\hline MIMO6_2 & 6 & 2 & SP3_2 & $16.66 \%$ \\
MIMO6_4 & 6 & 4 & SP3_4 & $8.33 \%$ \\
MIMO12_2 & 12 & 2 & SP6_2 & $12.5 \%$ \\
MIMO12_4 & 12 & 4 & SP6_4 & $6.25 \%$ \\
MIMO16_2 & 16 & 2 & SP8_2 & $8.33 \%$ \\
MIMO16_4 & 16 & 4 & SP8_4 & $4.16 \%$ \\
MIMO24_2 & 24 & 2 & SP12_2 & $4.16 \%$ \\
MIMO24_4 & 24 & 4 & SP12_4 & $2.08 \%$ \\
\hline \hline
\end{tabular}

$D_{\mathrm{X}}$ and $D_{\mathrm{Y}}$ are the separation of pilot bearing carriers in the frequency and time direction, respectively.

The I/Q polarization interleaving provides an additional diversity gain because each constellation symbol is transmitted over the two polarizations.

\section{3) Phase Hopping}

The phase hopping consists of a phase rotation to the symbols of the second transmit antenna. This may slightly degrade the performance under some specific channel conditions, but it improves overall performance by improving worst-channel phase correlated conditions [23].

The same phase hopping term from DVB-NGH [22] has been adopted for ATSC 3.0. The operation is mathematically expressed as:

$$
\left[\begin{array}{c}
S_{2 i}(\mathrm{Tx} 1) \\
S_{2 i+1}(\mathrm{Tx} 2)
\end{array}\right]=\left[\begin{array}{cc}
1 & 0 \\
0 & \mathrm{e}^{\mathrm{j} \phi(i)}
\end{array}\right]\left[\begin{array}{c}
Z_{2 i}(\mathrm{Tx} 1) \\
Z_{2 i+1}(\mathrm{Tx} 2)
\end{array}\right]
$$

where $\phi$ is the phase rotation angle, defined by the following equation:

$\phi(i)=\frac{2 \pi}{N} i,(N=9), i=0, \ldots, \frac{N_{\text {cells }}}{2}-1$

where $N_{\text {cells }}$ is the number of cells per FEC codeword. The phase rotation is initialized to $0^{\circ}$ at the beginning of each FEC block and is incremented by $2 \pi / 9$ for every cell pair.

\section{MIMO Pilots \\ 1) Pilot Schemes}

ATSC 3.0 defines twelve MIMO pilot schemes, four less than the number of SISO patterns. All MIMO patterns have equivalent SISO patterns, such that MIMO keeps the same Doppler and echo tolerance. This implies that the overall pilot density for MIMO is doubled so that the receiver is able to estimate the MIMO channel from each transmit antenna with the same accuracy in frequency and time.

Table II shows the parameters defining the MIMO scattered pilot patterns, the equivalent SISO pilot schemes, and the resulting pilot overhead. The terminology employed for the MIMO pilot patterns is MIMO $a \_b$, where $a=D_{\mathrm{X}}$ (separation of pilot carriers in the frequency direction) and $b=D_{\mathrm{Y}}$ (number of symbols forming one scattered pilot sequence, i.e., separation of pilot carriers in the time direction) per transmit antenna. It should be pointed out that the same restrictions for the MIMO pilot schemes in terms of the FFT and the GI apply as for SISO [14]. 
Fig. 4 illustrates two examples of MIMO scattered pilot schemes. Continual pilots and reserved carriers are not shown. In the figure, it can be seen that pilots are assigned to two pilot groups, namely group 1 and group 2. In MIMO the phases of the scattered, continual, edge, frame-start and frame-closing pilots may be modified compared to SISO, according to the MIMO pilot antenna encoding scheme. Two algorithms are defined for ATSC 3.0, namely:

- Walsh-Hadamard encoding.

- Null pilots encoding.

Only one pilot algorithm may be used in a given frame. Each algorithm applies a specific signal processing to each pilot group in each antenna, and they are briefly described next. The null pilots encoding might be more suited for static channel conditions (e.g., for fixed roof-top reception) while the WalshHadamard pilot encoding might be more suited for mobile channels. The reason is that the pilot spacing in time direction is increased to $2 D_{\mathrm{Y}}$ for the null pilots encoding compared to a pilot spacing of $D_{\mathrm{Y}}$ for the Walsh-Hadamard pilot encoding. For fast varying channel conditions, it is more challenging for the channel estimation to follow these channel variations if the pilot spacing in time direction is increased.

\section{2) Walsh-Hadamard Pilot Encoding}

With Walsh-Hadamard MIMO pilot encoding, the phases of the scattered, continual, edge, frame-start and frame closing pilots for group 2 are modified in the signal transmitted from antenna \#2. Both antennas shall transmit in all pilot positions. This scheme is used in DVB-T2 for MISO Alamouti [24], and it was also adopted in DVB-NGH [4].

The scattered pilots from antenna \#2 are inverted compared to antenna \#1 on alternate scattered pilot bearing carriers. The few continual pilots from transmitters in antenna \#2 falling on scattered-pilot-bearing carriers are inverted compared to antenna \#1 on carriers for which the scattered pilots are inverted, whereas continual pilots on non-scattered-pilotbearing carriers are not inverted. It should be noted that those cells which would be both a continual and a scattered pilot are treated as scattered pilots. Regarding the edge pilots antenna \#2, they are inverted compared to antenna $\# 1$ on odd numbered OFDM symbols. Finally, the frame start and boundary pilots from antenna \#2 are inverted compared to antenna \#1 on alternate scattered pilot bearing carriers.

\section{3) Null Pilots Encoding}

With MIMO null pilot encoding, the amplitudes of the scattered, continual, edge, frame-start and frame-closing pilots are modified in the signal transmitted from both transmit antennas \#1 and \#2. In opposition to Walsh-Hadamard encoding, with null pilots encoding each antenna transmits only on pilots belonging to one single group (either group 1 or group 2) with $3 \mathrm{~dB}$ increased transmit power to compensate for the null pilots not transmitted in the other group. The null pilots are inserted in the scattered pilots from antenna \#1 on alternate scattered-pilot-bearing carriers and OFDM symbols.

\section{Performance Simulation}

In this section we provide some illustrative physical layer performance simulation results about the overall performance of the MIMO scheme of ATSC 3.0, the use of non-uniform
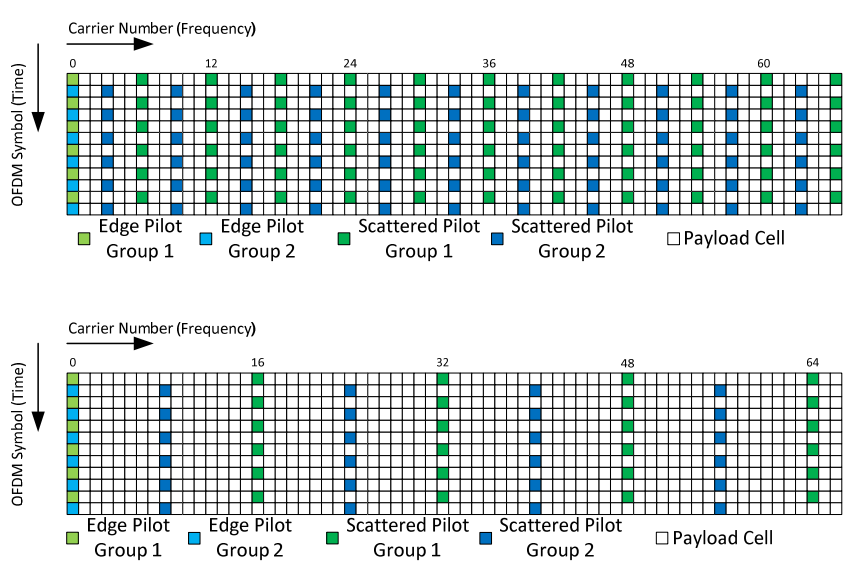

Fig. 4. Illustrations of the MIMO scattered pilot patterns: MIMO6 2 (top), and MIMO16_2 (bottom).

constellations for MIMO, and two of the signal processing algorithms of the MIMO precoder: antenna stream combination and phase hopping. Additional parameters are detailed next. A low-density parity-check (LDPC) code length of 64800 bits was used. Bit, time and frequency interleavers from the specification were also employed. The proposed criterion is to select the SNR that provides a bit error rate (BER) of $10^{-4}$.

\section{A. Overall Performance}

Fig. 5 shows the simulated MIMO performance of ATSC 3.0 compared to SISO for the mobile DVB-NGH channel [4] and the MGM fixed rooftop channel model [13]. The upper bounds of Fig. 1 are also shown for comparison. For the NGH mobile channel, QPSK, 16QAM and 64QAM constellations and a pilot pattern SP6_2 have been considered in the simulations. The considered constellations for the MGM fixed channel range from 64QAM up to 4096QAM (4kQAM) [18], and the pilot pattern used is SP12_4. For each constellation, four representative coding rates have been considered: $2 / 15,6 / 15$, 10/15 and 13/15 [25]. For MIMO, a maximum-likelihood (ML) demapper has been employed for simulations up to 64QAM and a minimum mean-square error (MMSE) demapper for higher order constellations in order to reduce the complexity burden. The equivalent pilot patterns for MIMO can be easily found in Table II.

In Fig. 5 it can be noted that the MIMO gains are larger for the fixed MGM channel compared to the NGH mobile channel. In the figure, we can also observe that the difference to the theoretical limit is higher for the MGM channel, especially at low SNRs. For instance, using $16 \mathrm{NUC}$ in each antenna, the difference to the theoretical limit is 3.6 and $7.9 \mathrm{~dB}$ for low and high CRs respectively. On the other hand, this difference becomes higher for the MGM channel, with 5.7 and $7.5 \mathrm{~dB}$ for low and high CRs respectively. In addition, the use of MMSE demapping implies a significant performance loss compared to the optimum ML. 

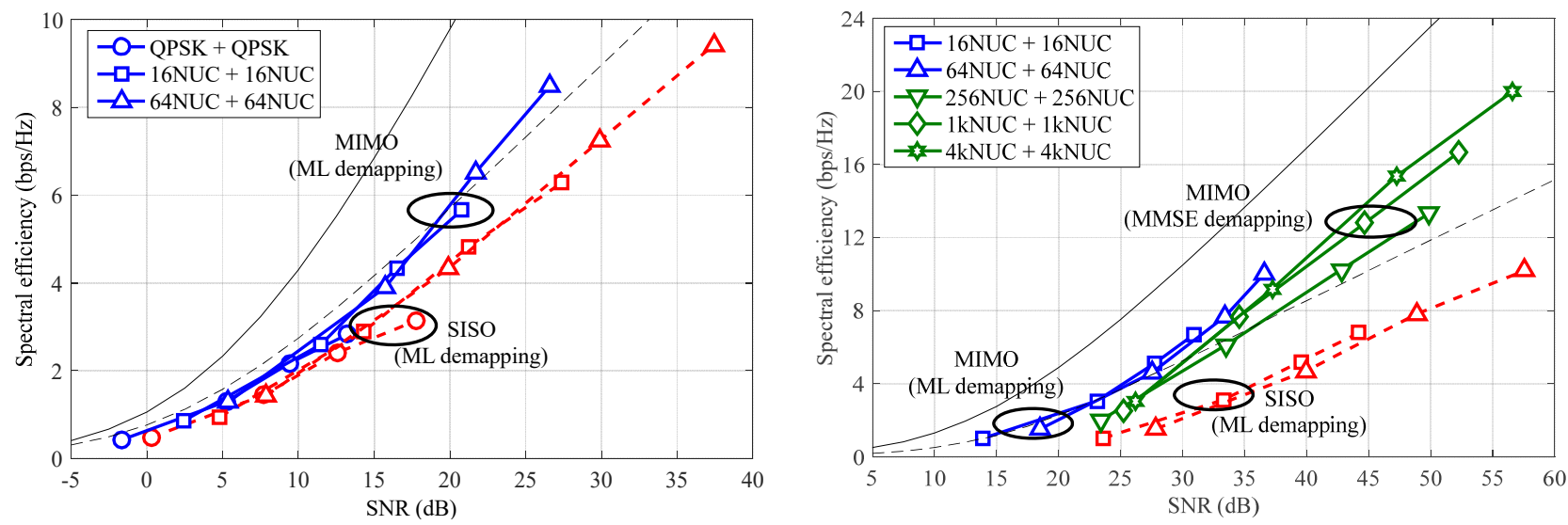

Fig. 5. MIMO performance and gain in ATSC 3.0 compared to SISO. Results presented for NGH mobile channel (left) and MGM channel (right).

MIMO channel depending on SNR and angle of departure: without phase hopping. System optimization would be possible only for a particular angle of arrival.

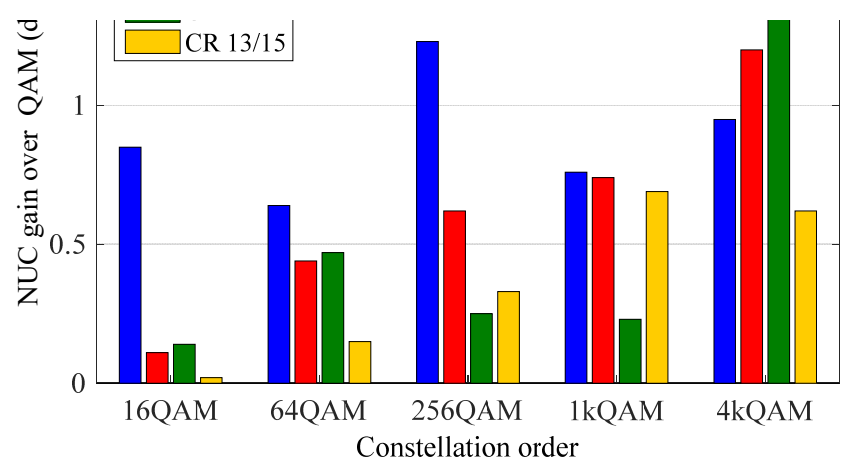

Fig. 6. Gain of non-uniform constellations compared to uniform constellations for MIMO.

\section{B. Non-Uniform Constellations for MIMO}

Fig. 6 depicts the SNR gain of NUCs over uniform QAM constellations for four representative coding rates $2 / 15,6 / 15$, $10 / 15$ and 13/15 in the MGM fixed rooftop channel model [13]. The MMSE demapper is used for all constellations in this case. High SNR gains are achieved especially for high constellation orders and low coding rates. However, it should be stressed that the constellations of ATSC 3.0 have been optimized for SISO [18], and hence the MIMO performance is not optimal. Further optimization may be possible e.g., considering a different SNR range and channel models.

\section{Precoder-Antenna Stream Combination}

Fig. 7 shows the SNR gain obtained with the rotation angles shown in Table I for QPSK and 16QAM in the NGH mobile channel [4] with a power imbalance of $9 \mathrm{~dB}$. The gain increases with the coding rate and low modulation orders, achieving a maximum SNR gain of up to $1.5 \mathrm{~dB}$ for QPSK 13/15. With a lower power imbalance, the selected angles still are optimum, but the gain decreases. For instance, the obtained gain with QPSK $13 / 15$ is reduced to $0.63 \mathrm{~dB}$ and $0.2 \mathrm{~dB}$ with power imbalances of $6 \mathrm{~dB}$ and $3 \mathrm{~dB}$ respectively. As mentioned in Section III, if there is no power imbalance there is no SNR gain due to the antenna stream combination, and the optimum rotation angle is $0 \mathrm{~dB}$ for all cases.

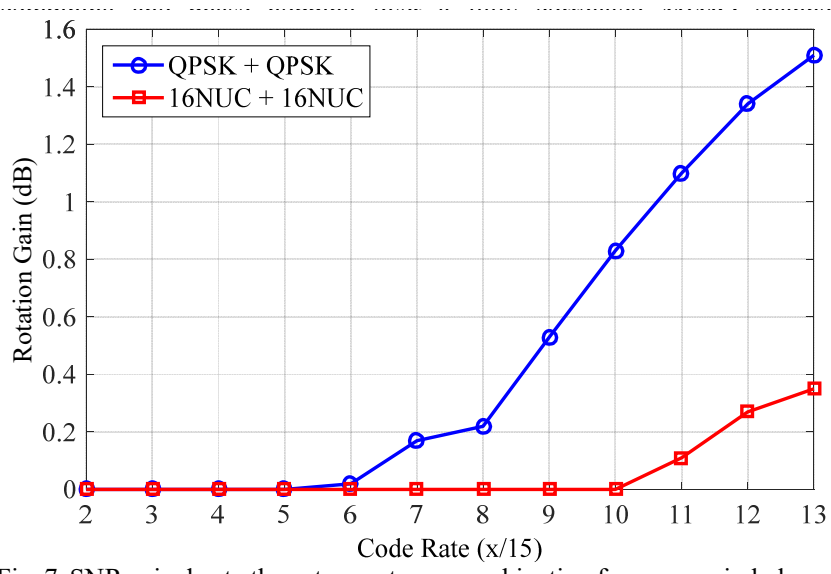

Fig. 7. SNR gain due to the antenna stream combination for a power imbalance of $9 \mathrm{~dB}$. NGH mobile channel mode for QPSK and 16QAM.

\section{Precoder - Phase Hopping}

Fig. 8 shows the BER performance of $(2 \times 2)$-sheer spatial multiplexing using non-uniform 16QAM with the baseline LDPC long code of code rate $9 / 15$ and corresponding bitinterleaver over a fully correlated MIMO channel representing worst case line-of sight channel conditions. Here, the channel matrix is modelled as:

$\boldsymbol{H}=\left[\begin{array}{ll}1 & 1 \\ 1 & 1\end{array}\right]\left[\begin{array}{cc}1 & 0 \\ 0 & e^{j \Phi_{t}}\end{array}\right]$

The underlying assumptions for this model are uniform linear antenna arrays at transmitter and receiver with planar wave propagation in-between. The angle of departure from the transmitter is denoted by $\Phi_{t}$.

Fig. 8 illustrates the dependence of the system performance on the angle of departure (AOD), where particular angles, e.g., $\Phi_{t}=0^{\circ}$ and $\Phi_{t}=90^{\circ}$, prove to have a catastrophic effect. In the absence of spatial precoding and phase hopping this is due to the independence of the transmitted streams resulting in erased or severely attenuated constellation points at the receiver.

Apparent from Fig. 8 is also that optimization of spatial precoding for this scenario will be optimal only for a limited range of angles of departure and suboptimal for others. The remedy is the combination of spatial precoding and phase hopping and their effect on the performance is shown in Fig. 9. The simulation parameters are identical to those for sheer SM 
in Fig. 8, but in addition precoding with antenna stream combination and phase hopping is employed. It is evident that precoding provide sufficient means to spread a FEC codeword over both data streams in such a way that degenerate received constellation points are mitigated and that ultimately the performance is rendered independent from the angle of departure.

\section{CONCLUSIONS AND OUTLOOK}

This paper provides an overview of the optional MIMO cross-polarized MIMO scheme adopted in ATSC 3.0. The MIMO scheme of ATSC 3.0 re-uses as much as possible the ATSC 3.0 SISO baseline specification, and it has been defined such that it is not outperformed by the SISO baseline in any aspects (e.g., time interleaving trade-off and echo resilience and Doppler performance). The MIMO scheme is very flexible, with several signal processing algorithms possible for the precoder (antenna stream combining, I/Q polarization interleaving, and phase hopping), the MIMO pilot encoding scheme (Walsh-Hadamard and null pilots), and with twelve different scattered pilot patterns. ATSC 3.0 also introduces the use of MIMO with non-uniform constellations, improving the transmission robustness compared to the use of uniform constellations.

MIMO is a promising technique to overcome the capacity limit of single antenna communications, and allows for terrestrial broadcasting to transmit two different data streams in the same radio frequency channel using simultaneously both horizontal and vertical polarizations. However, implementation requires important modifications at existing transmitter and fixed rooftop reception installations, and significantly more advanced receivers. Also, for low SNR regions there is smaller MIMO capacity gain compared to the high SNR region characteristic of fixed rooftop reception, although the relative gain at low SNR is very significant.

Nevertheless, in the U.S. MIMO can actually provide a larger comparative gain because the regulation allows to increase the total transmit power by transmitting the nominal transmit power in each polarization. Hence, in addition to the spatial multiplexing gain, MIMO can exploit a $3 \mathrm{~dB}$ power gain. MIMO is also an effective way to impose the use of two antennas at the receivers, which on average results in another 3 $\mathrm{dB}$ gain compared to SISO for cross-polarized transmissions. Also, the use of channel bonding will pave the way towards the implementation of MIMO receivers because it requires two tuners and support of twice the peak data rate, like MIMO but in two RF channels. For all these reasons, MIMO may become a key technology for ATSC 3.0, also taking into account that the current commercial state-of-the-art digital terrestrial television standard DVB-T2 does not include this feature.

\section{NOTE}

It is noted that that some items referred to in this paper, in particular, the rotation angles of the antenna stream combiner module of the MIMO precoder, could be changed during the candidate standard phase of the ATSC 3.0 physical layer specification.

\section{REFERENCES}

[1] A. Goldsmith, S. A., Jafar, N. Jindal, and S. Vishwanath, "Capacity limits of MIMO channels," IEEE Journal on Selected Areas on Communications, vol. 21, no. 5, pp. 684-702, June 2003.

[2] D. Tse and P. Viswanath, "Fundamentals of Wireless Communications," Cambridge University Press, 2005

[3] P. Moss, "2-by-2 MIMO Fixed Reception Channel Model for Dual-Polar Terrestrial Transmission," White Paper, British Broadcasting Corporation, 2008

[4] P. Moss and T. Y. Poon, "Overview of the Multiple-Input Multiple-Output Terrestrial Profile of DVB-NGH," in Next Generation Mobile Broadcasting, D. Gomez-Barquero, Ed. Boca Raton, FL, USA: CRC Press, 2013, pp. 549-580.

[5] D. Vargas, et al, "A MIMO-Channel-Precoding Scheme for Next Generation Terrestrial Broadcast TV Systems," IEEE Trans. Broadcast., vol. 61, no. 3, pp. 445-456, Sept. 2015

[6] J. Boyer, et al., "MIMO for Broadcast-Results from a High-Power UK Trial," White Paper, British Broadcasting Corporation, 2007.

[7] S. Saito, et al., "8K Terrestrial Transmission Field Tests Using Dualpolarized MIMO and Higher-order Modulation OFDM," IEEE Trans. Broadcast., vol. 62, no. 1, 2016

[8] D. Gomez-Barquero, C. Douillard, P. Moss, and V. Mignone, "DVBNGH: The Next Generation of Digital Broadcast Services to Handheld Devices," IEEE Trans. Broadcast., vol. 60, no. 2, pp. 246-257, June 2014.

[9] D. Vargas, D. Gozalvez, D. Gomez-Barquero, and N. Cardona, "MIMO for DVB-NGH, the Next Generation Mobile TV Broadcasting," IEEE Communications Magazine, vol. 51, no. 7, pp. 130-137, July 2013.

[10] L. Fay, L. Michael, D. Gomez-Barquero, N. Ammar, and M. W. Caldwell, "An Overview of the ATSC 3.0 Physical Layer Specification," IEEE Trans. Broadcast., vol. 62, no. 1, 2016.

[11] I. Eizmendi, et al., "DVB-T2: The Second Generation of Terrestrial Digital Video Broadcasting System," IEEE Trans. Broadcast., vol. 60, no. 2, pp. 258-271, June 2014.

[12] D. Gomez-Barquero and M. W. Caldwell, "Broadcast Television Spectrum Incentive Auctions in the U.S.: Trends, Challenges and Opportunities," IEEE Communications Magazine, vol. 53, no. 7, pp. 5056, July 2015.

[13] P. Moss, "MIMO Technology in Broadcasting - and an Application in Programme-Making," Proc. IEEE Broadcast Technology Society Gold Workshop on Next Generation Broadcasting, Cagliari, March 2013.

[14] B. Varadarajan and J. R. Barry, "The Outage Capacity of Linear SpaceTime Codes," IEEE Trans. Wireless Commun., vol. 4, no. 6, pp. 26422648, Nov. 2005.

[15] J.-S. Baek and J.-S. Seo, "Efficient Pilot Patterns and Channel Estimations for MIMO-OFDM Systems," IEEE Trans. Broadcast., vol. 58, no. 4, pp. 648-653, Dec. 2012.

[16] D. Perez-Calderon, V. Baena-Lecuyer, J. Chavez, A. C. Oria, and J. G. Doblado, "Simplified Detection for DVB-NGH MIMO Decoders," IEEE Trans. Broadcast., vol. 61, no. 1, pp. 84-90, 2015.

[17] L. Stadelmeier, D. Schneider, J. Zollner, and J. J. Gimenez, "Channel Bonding for ATSC 3.0," IEEE Trans. Broadcast., vol. 62, no. 1, 2016.

[18] N. Loghin, et al., "Non-Uniform Constellations for ATSC 3.0," IEEE Trans. Broadcast., vol. 62, no. 1, 2016.

[19] D. He, et al., "System Discovery and Signaling Transmission Using Bootstrap in ATSC 3.0," IEEE Trans. Broadcast., vol. 62, no. 1, 2016.

[20] S.-I. Park, et. al, "Low Complexity Layered Division Multiplexing System for ATSC 3.0," IEEE Trans. Broadcast., vol. 62, no. 1, 2016.

[21] S. Lopresto, R. Citta, D. Vargas, and D. Gomez-Barquero, "Transmit Diversity Code Filter Sets (TDCFS), a MISO Antenna Frequency PreDistortion Scheme for ATSC 3.0," IEEE Trans. Broadcast., vol. 62, no. $1,2016$.

[22] D. Vargas, S. Moon, W.-S. Ko, and D. Gomez-Barquero, "Enhanced MIMO Spatial Multiplexing with Phase Hopping for DVB-NGH," in Next Generation Mobile Broadcasting, D. Gomez-Barquero, Ed. Boca Raton, FL, USA: CRC Press, 2013, pp. 609-634.

[23] J. Jang, D. H. Kim, and Y. H. Lee, "Open-Loop Precoder for Spatial Multiplexing in MIMO Channels with Phase Correlation," IET Electronics Letters, vol. 50, no. 23, pp. 1766-1768, Nov. 2014,

[24] J. Mogarde, et al. "SFN-SISO and SFN-MISO Gain Performance Analysis for DVB-T2 Network Planning," IEEE Trans. Broadcast., vol. 60, no. 2, pp. 272-286, 2014.

[25] L. Michael and D. Gomez-Barquero, "Bit Interleaved Coding and Modulation for ATSC 3.0," IEEE Trans. Broadcast., vol. 62, no. 1, 2016. 
[26] L. Jiang, et al., "Correlation analysis of multiple-input multiple-output channels with cross-polarized antennas," in Proc. 14th Asia-Pac. Conf. Commun. (APCC), Tokyo, Japan, 2008, pp. 1-5.

David Gómez-Barquero received the double M.Sc. degrees in telecommunications engineering from the Universitat Politecnica de Valencia (UPV), Spain, and the University of Gävle, Sweden, in 2004, the Ph.D. degree in telecommunications from the UPV in 2009; and he carried out a 2-year post-doc at the Fraunhofer Heinrich Hertz Institute, Germany. He is a Senior Researcher (Ramon \& Cajal Fellow) with the Institute of Telecommunications and Multimedia Applications, UPV, where he leads a research group working on next generation broadcasting technologies. Previously, he hold visiting research appointments at Ericsson Eurolab, Germany, the Royal Institute of Technology, Sweden, the University of Turku, Finland, the Technical University of Braunschweig, Germany, the Sergio Arboleda University of Bogota, Colombia, and the New Jersey Institute of Technology, USA.

Dr. Gómez-Barquero has been since 2008 actively participating in the European digital television standardization forum DVB in different topics such as upper layer forward error correction, DVB-T2, T2-Lite, and DVB-NGH. In 2013, he joined the U.S. digital television standardization forum ATSC to work on ATSC 3.0, acting as Vice-Chairman of the Modulation and Coding Ad-Hoc Group. He is the Editor of the book entitled Next Generation Mobile Broadcasting (CRC Press, 2013).

David Vargas received his M.Sc. degree in Telecommunication engineering from Universitat Politecnica de Valencia (UPV), Spain in 2009. During his studies he spent one year at the University of Turku, Finland. Currently he is pursuing a Ph.D. degree at the Mobile Communications Group at the Institute of Telecommunications and Multimedia Applications (iTEAM), UPV. He has been a guest researcher in the summer of 2011 at the Vienna University of Technology, Austria, during 2013 at McGill University, Montreal, Canada, and a research intern in 2015 at BBC Research \& Development, London, UK. He has participated in the standardization process of the next generation mobile broadcasting standard DVB-NGH and is currently an active participant in the standardization process of the next-generation terrestrial broadcasting standard ATSC 3.0. His research interests include multi-antenna communications, signal processing for communications, and digital broadcasting.

Manuel Fuentes received his M.Sc. degree in telecommunication engineering and a second M.Sc. degree in communication technologies, systems and networks from the Universitat Politecnica de Valencia, Spain, in 2012 and 2013, respectively. Currently, he is pursuing a Ph.D. degree in telecommunications at the Institute of Telecommunications and Multimedia Applications (iTEAM), where he is working since 2012. He participated in several R\&D projects where his research interests were focused on protection ratio measurements and network planning activities between digital broadcasting and $4 \mathrm{G}$ (LTE) technologies. He is a current member of the DVB European forum and the ATSC forum, contributing to the ATSC 3.0 standardization process. His current research interests include innovative techniques in bitinterleaved coding and modulation systems, such as nonuniform constellations or signal space diversity techniques, and multi-antenna communications.

Peter Klenner received the Diploma and $\mathrm{PhD}$ degree in Electrical Engineering and Communication and Information Technology from the University of Bremen in 2004 and 2011, respectively. Since 2011, he is working as a Research Engineer with the Panasonic AVC Development Centre in Langen, close to Frankfurt a. M., Germany. His research interests include but are not limited to optimization, coding theory, iterative decoding, OFDM, MIMO communication, and massively parallel programming.

Sangchul Moon received his M.Sc. degree in Electrical Engineering from Korea Advanced Institute of Science and Technology (KAIST), in 2003. He is currently a principal research engineer in LG electronics. He has been involved in developing OFDM baseband demodulators, including DAB, DVB-T, DVB-T2. He has participated in the standardization process of the next generation broadcasting standards, DVB$\mathrm{T} 2 / \mathrm{C} 2 / \mathrm{NGH}$ and is currently an active participant in the standardization process of the next-generation terrestrial broadcasting standard ATSC 3.0. His research interests include multi-antenna communications, signal processing for communications, LDPC and digital broadcasting.

Jin-Yong Choi received the B.S., M.S. and Ph.D. degrees in electronics engineering from Yonsei University, Korea in 2004, 2007 and 2012, respectively. Since 2012, he has been with the Advanced Broadcasting \& Media Department, Advanced Standard R\&D Lab., LG Electronics (LGE), where he is a senior researcher engineer. His main research interests include signal processing and MIMO systems for providing highquality communication and broadcasting services, i.e., HDTV, UHDTV, and mobile TV.

Daniel Schneider received the Dipl.-Ing. degree in electrical engineering (with main focus on signal processing and communications) and the Dr.-Ing. degree for his thesis "Inhome power line communications using multiple input multiple output principles" from the University of Stuttgart, Stuttgart, Germany, in 2006 and 2012, respectively. He is currently a Senior Engineer at the European Technology Center, Sony, Stuttgart, where his work is concerned with communications systems and signal processing.

Kenichi Murayama received his M. Eng. degree in Mechanical Engineering from Niigata University, Japan in 1996. He joined NHK (Japan Broadcasting Corporation) in 2002. From 2002 to 2008, he has worked at the NHK Engineering Administration Department / Transmission \& Audience Reception Engineering Center. From 2008 to 2013, he has worked at the Advanced Transmission Systems Research Division in NHK Science and Technology Research Laboratories (STRL) and has been engaged in research and development related to next generation of digital terrestrial broadcasting. 